\section{P1-538 LIFE-COURSE DETERMINANTS OF DISSATISFACTION WITH THE DENTAL APPEARANCE AT AGE 24}

doi:10.1136/jech.2011.142976h.26

${ }^{1} \mathrm{~F}$ Vargas-Ferreira, ${ }^{2} \mathrm{~K}$ Peres, ${ }^{2} \mathrm{M}$ Peres, ${ }^{1} \mathrm{~B}$ Horta, ${ }^{1} \mathrm{D}$ Gigante, ${ }^{1} \mathrm{~F}$ Demarco. ${ }^{1}$ Federal University of Pelotas, Pelotas, Rio Grande do Sul, Brazil; ${ }^{2}$ Federal University of Santa Catarina, Florianopólis, Santa Catarina, Brazil

Introduction Dental appearance comprises an important aspect of oral health. However, no study has investigated the dynamics of dissatisfaction with dental appearance and other aspects of oral health using a birth cohort study.

Objectives This study estimated the prevalence of dissatisfaction with the dental appearance among 24-yr-old Brazilian adults and the association with potential life course risk factors. A crosssectional study nested in a birth cohort study was carried out in Pelotas, Brazil. A representative sample $(n=720)$ of all 5914 births occurring in Pelotas in 1982 was prospectively investigated and the outcome was assessed in 2006. Exploratory variables were collected at birth, at 15 and $24 \mathrm{yr}$ of age and included demographic/socioeconomic, oral health, appearance satisfaction and use of dental services. Dissatisfaction with dental appearance at aged $24 \mathrm{yr}$ was the outcome. Unadjusted and adjusted multivariable Poisson regression was performed followed a theoretical model.

Results The prevalence of the outcome was $43.5 \%$ (39.8;47.2). In the final model, low socio-economic status through the life-course [RR 1.21 (95\% CI 1.00 to 1.57$)$ ], malocclusion at aged $15 \mathrm{yr}$ [RR 1.34 (1.13 to 1.59)], dental pain at aged $24 \mathrm{yr}$ [RR 1.29 (1.08 to 1.55)], adolescent's dissatisfaction with appearance [RR 1.20 (1.01 to 1.43)], and untreated dental caries at aged 24 (highest tertile) [RR 1.82 (1.46 to 2.27)] were associated with dental appearance dissatisfaction.

Conclusion Our findings showed that the individuals with dissatisfaction with their physical appearance at age 15 were more likely to rate their oral health as worse than their counterparts. Participants with downwardly income trajectory had the worst dissatisfaction with the dental appearance.

\section{P1-539 ALCOHOL USE AMONG ADOLESCENTS IN GUADALAJARA, MEXICO: PATTERNS AND ASSOCIATED FACTORS}

doi:10.1136/jech.2011.142976h.27

M G Vega-Lopez,* G J Gonzalez-perez, C E Cabrera-pivaral. University of guadalajara, Guadalajara, Mexico

Introduction This study describes the characteristics of alcohol consumption among adolescents in Guadalajara, Mexico and identifies factors associated with alcohol use.

Methods A multistage random sample, representative of the student population at the secondary level in public schools in Guadalajara, Mexico, during 2009-2010 was designed. It included seven high schools and 716 students, aged between 11 and 16 . Multivariate analysis was conducted using a logistic regression model, to identify the association between socioeconomic factors and alcohol intake.

Results $61 \%$ of students reported having ever consumed alcohol, and almost a third had their first alcohol consumption before age 11. A quarter of those who currently use alcohol consume four drinks or more. $5 \%$ of students admit to having come to school on occasion under the influence of alcohol. Factors such as being beaten by parents or older siblings or to be a woman are significantly associated to alcohol consumption.

Conclusions The main factors associated with alcohol consumption among students are gender and family dynamics. Being a woman increases $40 \%$ probability of consuming alcoholic beverages but being a victim of domestic violence increased by more than $50 \%$ probability to consume alcohol. Although the social imaginary are believed to friends who lead the teenagers to drink alcohol, the results show that it is in the family environment where the initial phase of experimentation is developed.

\section{P1-540 WITHDRAWN}

\section{P1-541 REPORTING OF ELIGIBILITY CRITERIA OF RANDOMISED TRIALS: EMPIRICAL STUDY COMPARING TRIAL PROTOCOLS TO SUBSEOUENT ARTICLES}

doi:10.1136/jech.2011.142976h.28

${ }^{1}$ A Bluemle, ${ }^{1,2} \mathrm{~J}$ Meerpohl, ${ }^{1} \mathrm{G}$ Ruecker, ${ }^{1} \mathrm{G}$ Antes, ${ }^{1} \mathrm{M}$ Schumacher, ${ }^{1,3} \mathrm{E}$ von Elm. ${ }^{*}$ ${ }^{1}$ German Cochrane Center, Department of Medical Biometry and Statistics, Institute of Medical Biometry and Medical Informatics, University Medical Center, Freiburg, Germany; ${ }^{2}$ Division of Pediatric Hematology \& Oncology, Department of Pediatrics, University Medical Center, Freiburg, Germany; ${ }^{3}$ Swiss Paraplegic Research, Nottwil, Switzerland

Introduction Accurate and complete reporting of information about trial participants is important to apply results in clinical practice. We studied whether and how eligibility criteria (EC) of participants of randomised trials pre-specified in study protocols are reported in subsequent articles.

Methods By electronic literature searches and survey of applicants, we identified 78 full publications corresponding to 52 trial protocols submitted in 2000 to the ethics committee of the University of Freiburg/ Germany. From both sources we extracted information on EC, whether they were matching, missing, modified or added in the publications.

Results We found differences between protocols and publications for all 52 trials. Information on $\mathrm{EC}$ was missing in publications corresponding to 52 trials (100\%; $95 \%$ CI 93 to 100), modified for 44 trials (85\%; $95 \%$ CI 72 to 93 ) or newly added for 21 trials (41\%; $95 \%$ CI 27 to 55). The mean number of EC per trial was 25 (range 7-43); the mean proportion of matching EC per trial was 50\% (range $13-93$; $95 \%$ CI 44 to 55). Of $1248 \mathrm{EC}$ pre-specified in protocols, 606 (49\%) were matching, 479 (38\%) missing, and 163 (13\%) modified in subsequent publications. Fifty-one EC were added to publications. Most of the missing EC (96\%) and about half of the modified EC (54\%) suggested broader study populations, most added EC (86\%) narrower study populations.

Conclusion Published trial reports generally do not reflect the definitions of study populations pre-specified in the protocol. This hampers a proper assessment of the applicability of trial results.

\section{P1-542 FEASIBILITY, ACCEPTABILITY AND UTILITY OF AN E-CARDIOVASCULAR RISK PLATFORM AMONG PHYSICIANS AND PATIENTS IN THE PRIMARY CARE SETTING}

doi:10.1136/jech.2011.142976h.29

${ }^{1} \mathrm{~J} \mathrm{M}$ Johnston, ${ }^{1} \mathrm{M} T \mathrm{Vu},{ }^{*}{ }^{1} \mathrm{M} \mathrm{C}$ Schooling, ${ }^{1} \mathrm{H}$ Tinsley, ${ }^{1} \mathrm{~A}$ K L Chan, ${ }^{2} \mathrm{~J} \mathrm{H}$ B Kong, ${ }^{3} \mathrm{~L}$ C Y Tsang, ${ }^{4} \mathrm{~J}$ Chan. ${ }^{1}$ Department of Community Medicine and School of Public Health, Li Ka Shing Faculty of Medicine. The University of Hong Kong, Hong Kong; ${ }^{2}$ Central Medical Practice, Hong Kong; ${ }^{3}$ Department of Health, The Government of the Hong Kong Special Administrative Region, Hong Kong SAR, People's Republic of China, Hong Kong: ${ }^{4}$ Queen Elizabeth Hospital, Hong Kong SAR, People's Republic of China, Hong Kong

Introduction Cardiovascular disease (CVD) is the leading cause of death in developed countries. Individually-tailored CVD risk reduction (CVDRR) decision support tools delivered through hand-held devices (e-platform on mobile phone) may improve provider planning for and patient involvement with CVDRR strategies. We conducted a pilot study to assess provider and patient attitudes towards, as well as the feasibility, utility, and acceptability of e-platform technologies via a mobile phone for CVDRR in primary care.

Methods 20 patients, aged 45-79 years, using a JAVA enabled and internet accessible handset were recruited from two private and one 